\title{
DESARROLLO MORFOGÉNICO EN MICROPROPAGACIÓN DE SEMILLAS DE Eucalyptus nitens MEJORADAS GENETICAMENTE
}

\author{
Obando Camino, Maritza ${ }^{3}$, Matamala, Felipe ${ }^{4}$, Carmona, Juan Carlos ${ }^{5}$
}

\section{RESUMEN}

El mejoramiento genético de Eucalyptus nitens requiere material genético de alto valor. El establecimiento de plantaciones clonales requiere de una propagación rápida, donde el enraizamiento es una característica de recalcitrancia.

Esta investigación analizó respuestas morfogénicas de semillas de cruzamientos controlados en micropropagación.

Se utilizó semillas de E. nitens de 6 cruzamientos controlados, para el control se utilizó mezcla familiar. Semillas estériles fueron sembradas en medio MS (1962) modificado, $100 \mathrm{mg} / \mathrm{L}$ inositol, 2\% sacarosa, 0,7\% agar, $\mathrm{pH} 5,7$.

Para la inducción de brotes múltiples y elongación utilizó medio MS (1962) modificado, $0.25 \mathrm{mg} / \mathrm{L}$ BAP y $0,01 \mathrm{mg} / \mathrm{L}$ ANA. El enraizamiento se realizó en medio MS (1962) modificado, con regulador hormonal. Las vitroplántulas fueron mantenidas a $23^{\circ} \mathrm{C}$, fotoperiodo $16 / 8 \mathrm{~h}, 25 \mu \mathrm{molm}$ ${ }^{2}$ seg $^{-1}$.

Para 203 clones establecidos de E. nitens, un 69\% de ellos desarrollaron brotes múltiples, en 4 ciclos de subcultivo. En las semillas control se diferenciaron 32 clones, donde, un $86,5 \%$ desarrolló brotes múltiples.

En el cruzamiento A se diferenciaron 26 clones con $100 \%$ de multiplicación, para el cruzamiento $\mathrm{F}$ se multiplicaron $36 \%$ de los clones. De 89 clones diferenciados en semillas de cruzamientos, la cruza B obtuvo un $60 \%$ de enraizamiento y un $51 \%$ en la cruza $\mathrm{E}$, para 32 clones control solo el $29 \%$ enraizaron.

Los resultados mostraron que no existieron diferencias significativas en multiplicación, entre las semillas mejoradas y control. Existieron diferencias significativas en enraizamiento entre las cruzas $\mathrm{B}$ y $\mathrm{E}$ en relación al control.

El análisis de resultados se efectuón con ANOVA de una vía con 95\% de confianza, Test de Tukey.05 y STATISTICA versión 6.0. Para graficar se utilizó GraphPad Prism 5.

Palabras claves: Micropropagación, enraizamiento, Eucalyptus nitens.

3 Universidad Arturo Prat, Concepción, Chile, maritzaobando@unap.cl

${ }^{4}$ Universidad de Concepción, sede Los Angeles, Chile. felipematamala@udec.cl

${ }^{5}$ Sponte Sua, Concepción, Chile.carmona.juancarlos@gmail.com 


\section{SUMMARY}

The breeding of Eucalyptus nitens needs high value genetic seeds. The establishment of clonal plantations requires a fast propagation, where the rooting is a feature of recalcitrance.

This research studied morphogenic answers of seeds from controlled crosses in micropropagation.

E. nitens seeds from 6 controlled crosses were utilized and the control was from a family mix. Sterile seeds were cultivated in modified MS medium (1962), $100 \mathrm{mg} / \mathrm{L}$ myo-inositol, $2 \%$ sucrose, $0.7 \%$ agar, pH 5.7 .

The multiple shoots and elongation induction was realized in modified MS medium (1962), $0.25 \mathrm{mg} / \mathrm{L}$ BAP and $0.01 \mathrm{mg} / \mathrm{L}$ ANA. Rooting was developed in modified MS medium (1962), with growth regulator. Shoots were maintained at $23^{\circ} \mathrm{C}, 16 / 8 \mathrm{~h}$ photoperiod, $25 \mu \mathrm{molm}^{-2} \mathrm{seg}^{-1}$.

For 203 E. nitens clones, 69\% of them differentiated multiple shoots in 4 culture cycles. In control seeds were differentiated 32 clones, where a $86,5 \%$ developed multiple shoots. The A crosse differentiated 26 clones with $100 \%$ of multiplication, by $\mathrm{F}$ crosse were obtained $36 \%$ of clones in multiplication. Of 89 clones differentiated from crosses, the B crosse obtained $60 \%$ of rooting and $\mathrm{E}$ crosse $51 \%$, in 32 control clones only $29 \%$ registered rooting.

Results showed that there were no significant differences in multiplication between breeding seeds and control. Significant differences were found between B and E crosses rooting in relation to the control.

Results were analyzed with one way ANOVA with a 95\% of confidence level, Tukey Test (.05) and STATISTICA version 6.0. Graphics were made with Graph Pad Prism 5.

Keywords: Micropropagation, rooting, Eucalyptus nitens. 


\section{INTRODUCCIÓN}

Las especies de Eucalyptus son ampliamente empleadas en plantaciones a nivel mundial. Son especies originarias de Australia y se las planta en un rango amplio de condiciones ambientales, desde el nivel del mar a zonas precordilleranas y desde altas pluviometrias a zonas semiáridas. Varían en forma, desde arbustos a grandes árboles (Williams y Woinaski, 1997). Las 10 más importantes especies de Eucalyptus plantadas a nivel mundial incluyen a $E$. grandis, $E$. camaldulensis, E. tereticornis, E. globulus, E urophylla, E. viminalis, E. saligna, E. deglupta, E. excerta y luego E. citridiora, E. paniculata, y de ellas las cuatro primeras resultan ser las más importantes a nivel industrial (Eldridge et al., 1993)

Hoy día se considera a Eucalyptus nitens como la especie latifoliada de mejor proyección en la industria forestal nacional, debido a su alto incremento volumétrico anual, que en sitios de alta productividad supera los $40 \mathrm{~m}^{3} /$ ha/año, con semillas de baja ganancia genética aún, y al hecho de que en algunos casos casi duplica el crecimiento de Pinus radiata y Eucalyptus globulus. Por otra parte, es una especie que tiene una alta adaptabilidad a climas fríos, constituyendo una alternativa de plantación para zonas donde no es posible plantar Eucalyptus globulus debido a esta limitante. Diferentes estudios han demostrado la aptitud de esta especie para los procesos industriales de secado, aserrado y producción de láminas. De esta forma, Chile está frente a una especie potencialmente interesante para la industria forestal.

Inicialmente las plantaciones de Eucalyptus nitens se han realizado en el país con plantas procedentes de semillas de árboles de Victoria y Nueva Gales del Sur de Australia o de áreas productoras de semillas establecidas en el país con semillas originales de Australia. El establecimiento de huertos semilleros ha permitido el abastecimiento de semilla mejorada genéticamente la cual ha sido utilizada para producción de plantas en pequeñas cantidades aún.

Se ha trabajado en la reproducción vegetativa de la especie, siendo su limitada capacidad de enraizamiento la mayor dificultad para esto, aspecto que ha afectado el establecimiento de plantas de genotipos élite propagadas vegetativamente. La utilización de técnicas de micropropagación logra incrementar la producción de plantas en un corto plazo para ser establecidas productivamente. La masificación de genotipos seleccionados de Eucalyptus nitens, se ha logrado a través de las técnicas de micropropagación, autores como Gomes y Canhoto (2003) utilizando como explantes iniciales ápices de plantas de 1 año han logrado el desarrollo de estas. Los programas de mejoramiento genético de la especie han permitido bajos volúmenes de semilla mejorada genéticamente, siendo necesario incrementar rápidamente la producción de plantas a través de micropropagación, Si bien la germinación de semillas de $E$. nitens es conocida, el desarrollo morfológico de las plantas in vitro se encuentra condicionado por las características nutricionales del medio de cultivo, reguladores hormonales utilizados, gelificante, adendas orgánicas y factores ambientales (Dutra et al., 2009).

\section{OBJETIVO}

El presente estudio tuvo como objetivo evaluar el desarrollo morfogénico en micropropagación de semillas mejoradas genéticamente en seis cruzamientos de E. nitens.

\section{MATERIAL Y MÉTODO}

El material vegetal fue semillas de $E$. nitens pertenecientes a 6 cruzamientos controlados las que fueron cultivadas in vitro. Como control se utilizaron semillas de $E$. nitens de mezcla familiar.

Se efectuó cultivo in vitro el año 2011, para ello las semillas fueron desinfectadas en una solución $20 \%$ hipoclorito de sodio en presencia de un surfactante por $20 \mathrm{~min}$, luego fueron lavadas en agua destilada por $3 \mathrm{~h}$, luego colocadas en matraz y desinfectadas en una solución $3 \%$ peróxido de hidrógeno por $10 \mathrm{~min}$, lavadas en agua destilada estéril y estratificadas a $4^{\circ} \mathrm{C}$ por $24 \mathrm{~h}$. 
Posteriormente, se las sembró en medio 0,7\% agar-agua, $2 \%$ sacarosa, $\mathrm{pH} 5,7$. Brotes germinados fueron subcultivados en medio de cultivo Murashige-Skoog (1962) con $500 \mathrm{mg} / \mathrm{L}$ glutamina, $100 \mathrm{mg} / \mathrm{L}$ inositol, $2 \%$ sacarosa, $\mathrm{pH} 5,7,0.7 \%$ agar.

Se incubó los explantes a $23^{\circ} \mathrm{C}$, fotoperiodo $16 / 8 \mathrm{~h}, 25 \mu \mathrm{molm}^{-2} \mathrm{seg}^{-1}$.

Para la multiplicación y elongación in vitro los brotes fueron cultivados en medio Murashige-Skoog (1962) con 1,1 $\mu \mathrm{M}$ BAP, $0.05 \mu \mathrm{M}$ ANA, 500 mg/L glutamina, $100 \mathrm{mg} / \mathrm{L}$ inositol, $2 \%$ sacarosa, $\mathrm{pH} 5,7,0,7 \%$ agar.

Para el enraizamiento in vitro los brotes elongados procedentes de semillas control fueron enraizados in vitro en medio $\mathrm{MS} / 4,10 \mathrm{mg} / \mathrm{L}$ pantotenato de calcio, concentraciones de 4,8714,7-24,37-39-58,5 $\mu \mathrm{M}$ AIB, $100 \mathrm{mg} / \mathrm{L}$ inositol, 1,2\% sacarosa, $\mathrm{pH}$ 5,7, 0,7\% agar.

Se incubó los explantes a $23^{\circ} \mathrm{C}$, fotoperiodo $16 / 8 \mathrm{~h}, 60 \mu \mathrm{molm}^{-2} \mathrm{seg}^{-1}$. Los brotes elongados de cruzamientos y control fueron enraizados in vitro en el medio base ya descrito con 39 $\mu \mathrm{M}$ AIB.

En el análisis estadístico el material genético fue evaluado in vitro en las etapas de germinación, multiplicación y enraizamiento.

Se analizó los resultados con ANOVA de una vía con 95\% de confianza, Test de Tukey $(\mathrm{P} \leq .05)$ y STATISTICA versión 6.0. Para graficar se utilizó GraphPad Prism 5.

\section{RESULTADOS}

Las semillas cultivadas in vitro mostraron similar respuestas germinativa para las cruzas Control, A, B, C, F.

Existieron diferencia significativa en el porcentaje de semillas germinadas en las cruzas $\mathrm{E}$ y $D$, en relación a las semillas control y demás cruzamientos. (Figura $N^{\circ} 1$ ).

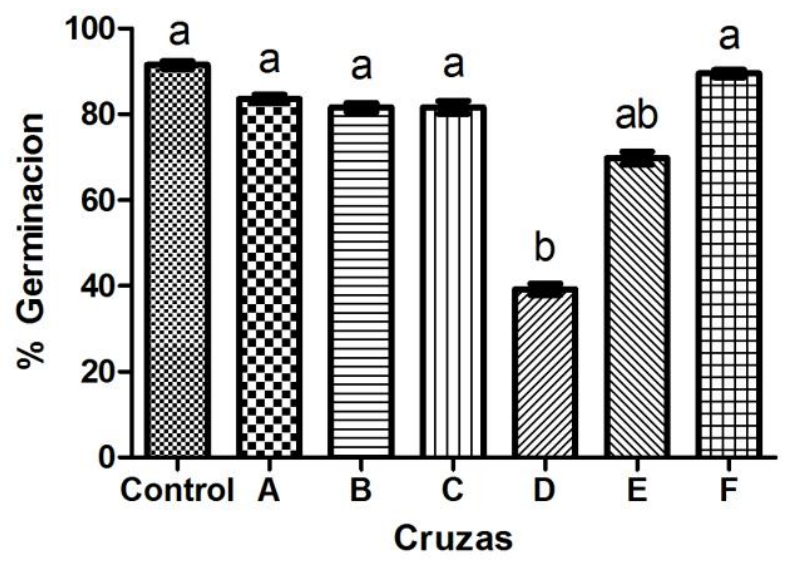

Barras verticales son error estándar.

Letras distintas indican diferencia significativa Tukey $(P \leq .05)$

Figura $\mathrm{N}^{\circ} 1$

PORCENTAJE DE GERMINACIÓN DE SEMILLAS CONTROL Y CRUZAMIENTOS 
Los brotes germinados permitieron el establecimiento in vitro de 203 clones, de ellos 140 clones iniciaron la etapa de multiplicación, existiendo 63 clones que no prosperaron en el medio de multiplicación establecido. (Cuadro $N^{\circ} 1$ )

Cuadro $\mathrm{N}^{\circ} 1$

NÚMERO DE SEMILLAS SEMBRADAS, CLONES ESTABLECIDOS Y EN MULTIPLICACIÓN EN SEMILLAS CONTROL Y CRUZAMIENTOS CONTROLADOS

\begin{tabular}{|c|c|c|c|}
\hline Cruzas & $\begin{array}{c}\text { Siembra } \\
\left(\mathbf{N}^{\circ} \text { Semillas }\right)\end{array}$ & $\begin{array}{c}\text { Establecimiento } \\
\left(\mathbf{N}^{\circ} \text { Clones }\right)\end{array}$ & $\begin{array}{c}\text { Multiplicación } \\
\left(\mathbf{N}^{\circ} \text { Clones con Brote }\right)\end{array}$ \\
\hline Control & 96 & 37 & 32 \\
A & 49 & 26 & 26 \\
B & 49 & 33 & 27 \\
C & 49 & 19 & 15 \\
D & 51 & 11 & 6 \\
E & 53 & 33 & 18 \\
F & 57 & 44 & 16 \\
\hline
\end{tabular}

Brotes elongados procedentes de semillas control fueron evaluadas en su respuesta de enraizamiento. Para ello, los explantes fueron cultivados en medio con concentraciones crecientes de AIB (mg/L), donde la dosis de $39 \mu \mathrm{M}$ AIB logró enraizar el $44,4 \%$ de los brotes establecidos en el medio de cultivo.

Existieron diferencias significativas en las respuestas de enraizamiento entre las concentraciones de $39 \mu \mathrm{M}$ AIB en relación a 4,87 $\mu \mathrm{M}$ y 14,7 $\mu \mathrm{M}$ AlB.

Concentraciones de $24,37 \mu \mathrm{M}$ y $58,5 \mu \mathrm{M}$ AIB no tuvieron diferencias significativas entre ellas. El experimento fue realizado dos veces (Figura 2).

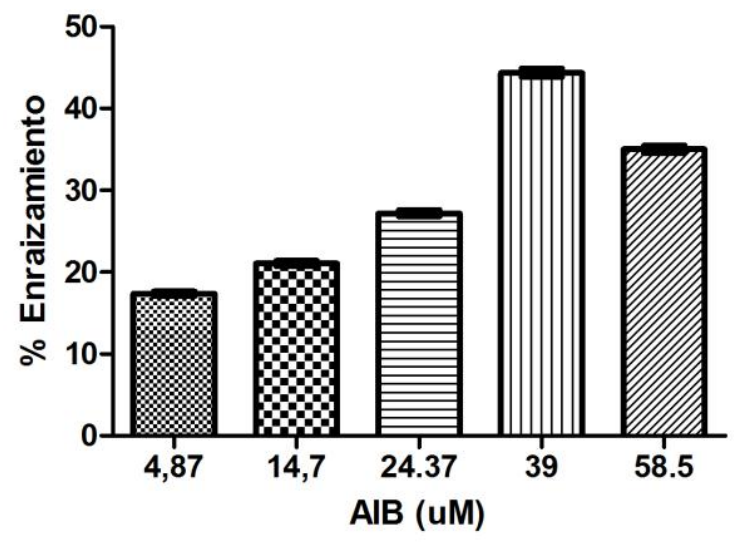

Barras verticales son error estándar.

Figura $N^{\circ} 2$

PORCENTAJE DE ENRAIZAMIENTO DE BROTES PROCEDENTES DE SEMILLAS CONTROL BAJO DIFERENTES CONCENTRACIONES DE AIB EN MEDIO DE CULTIVO

Las respuestas de diferenciación morfogénica de explantes de $E$. nitens se pueden apreciar en la Figura $\mathrm{N}^{\circ} 3$. 


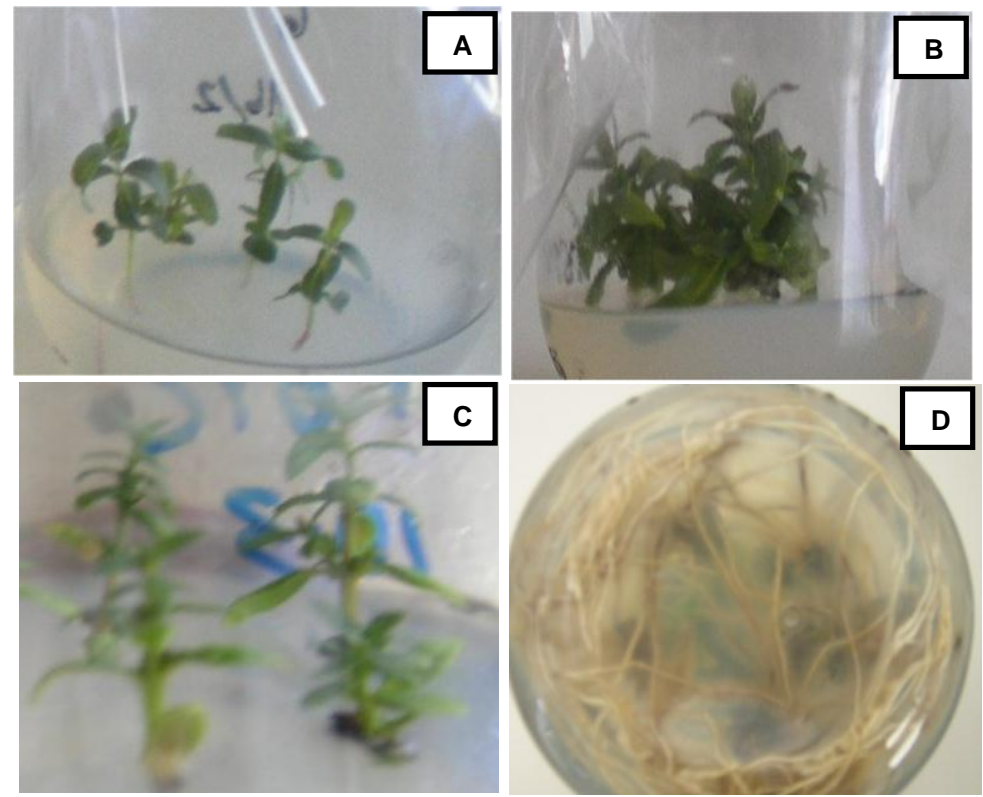

A: brotes en semillas germinadas B: brotes en etapa de multiplicación

$\mathrm{C}$ : brotes elongados $\mathrm{D}$ : desarrollo de raíces en brotes

Figura $\mathrm{N}^{\circ} 3$

ETAPAS DE DIFERENCIACIÓN MORFOGÉNICA DE SEMILLAS IN VITRO

Los clones de las diferentes cruzas fueron sometidos a 4 ciclos de multiplicación, posteriormente, los brotes elongados fueron cultivados en medio de enraizamiento con $39 \mu \mathrm{M} \mathrm{AIB}$. Se encontró diferencias significativas en las respuestas de enraizamiento entre los diferentes cruzamientos (Figura $\mathrm{N}^{\circ} 4$ ).

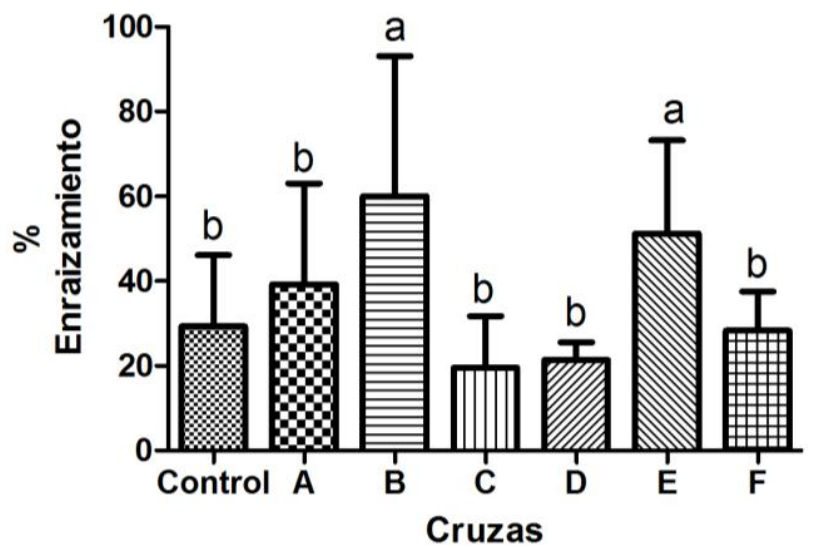

Barras verticales son error estándar.

Letras distintas indican diferencia significativa Tukey $(P \leq .05)$

Figura $N^{\circ} 4$

PORCENTAJE DE ENRAIZAMIENTO DE BROTES MICROPROPAGADOS

DESDE SEMILLA CONTROL Y CRUZAMIENTOS CONTROLADOS 
Los resultados de enraizamiento mostraron que las cruzas $B$ y $E$ tuvieron un porcentaje de enraizamiento de $60 \%$ y $51,2 \%$, respectivamente, existiendo diferencias significativas con los demás cruzamientos y control. En tanto no existieron diferencias significativas en enraizamiento entre los brotes procedentes de semillas control vs brotes de las cruzas A, C, D y F.

En la Figura $\mathrm{N}^{\circ} 5$ se aprecia el porcentaje de enraizamiento por clon, obtenido en plántulas del cruzamiento B (Figura 5).

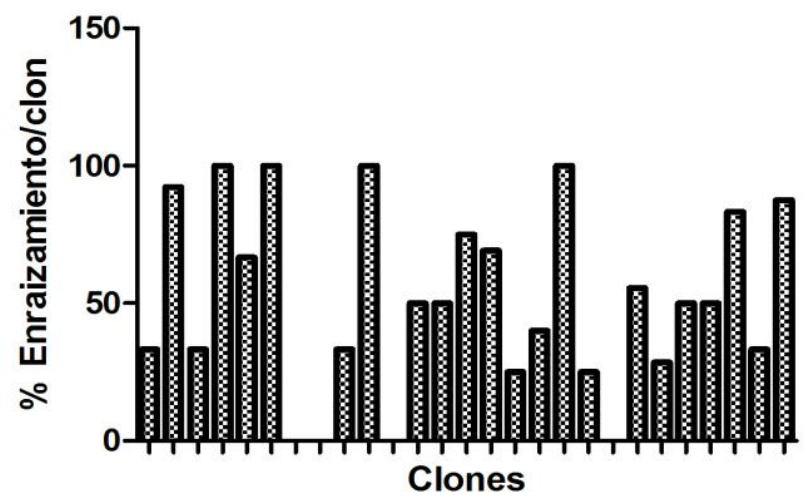

Figura $\mathrm{N}^{\circ} 5$

PORCENTAJE DE ENRAIZAMIENTO POR CLON EN PLÁNTULAS DE EL CRUZAMIENTO B MICROPROPAGADAS

Se observa en la figura que en clones de la cruza B existieron respuestas diferenciales de enraizamiento por clon; genotipos con $100 \%$ de brotes enraizados hasta clones con ausencia de enraizamiento bajo las condiciones in vitro ensayadas. El proceso de aclimatación de las plántulas se encuentra en desarrollo.

\section{DISCUSIÓN}

Hoy día existe una demanda de plantas con alto potencial de crecimiento, la propagación clonal a través de cultivo de tejidos tiene el potencial para entregar altas tas as de multiplicación de genotipos uniformes, resultando en producciones de menor tiempo. Las modificaciones realizadas a los medios de cultivo, dosis de reguladores hormonales, condiciones ambientales de la micropropagación, han colaborado en mejorar las respuestas rizogénicas en los genotipos seleccionados. Los programas de cruzamientos controlados en desarrollo constituyen la base para la obtención de semilla genéticamente mejorada, la cual requiere ser clonada y masificados los individuos a través de esta técnica.

El patrón de germinación de semillas de una especie se ve afectada por la fuente de semilla, la procedencia de esta, la familia, el estado nutricional parental, pretratamientos, madurez de la semilla, preacondicionamiento ambiental durante el desarrollo de la semilla y tamaño de la semilla (Chaisurisri et al., 1992).

Estudios en semillas de E. nitens de diferentes procedencias mostraron que las condiciones térmicas afectan la tasa de germinación de estas, donde a temperaturas entre $18^{\circ} \mathrm{C}$ a $25^{\circ} \mathrm{C}$ se logran las mejores tasas de germinación (Humara et al., 2000), condiciones similares a las de esta investigación, donde se logra un porcentaje de germinación de aproximadamente $90 \%$ en las semillas control, cruzas A, B, C y F, disminuyendo en las cruzas E y D (Figura $\mathrm{N}^{\circ} 1$ ). Los resultados indican que la alta germinación se logró utilizando en el ambiente una temperatura de $23^{\circ} \mathrm{C}$, siendo favorable para la mayor parte de las semillas cultivadas in vitro (Taylor et al., 1999). 
Del total de genotipos establecidos in vitro, 64 de ellos no lograron proliferar en la fase de multiplicación de brotes (Cuadro $N^{\circ} 1$ ), esto respondería a que esos genotipos podrían tener un desarrollo morfogénico adecuado bajo menores contenidos de macronutrientes en el medio de cultivo, lo cual sería corroborado por lo definido por Gomes y Canhoto (2003) quienes indicaron que la mejor tasa de multiplicación $(2,25)$ se obtuvo al utilizar macronutrientes a mitad de concentración en el medio MS (1962), junto a adendas orgánicas del medio De Fossard (De Fossard, 1974). Por otra lado, la utilización de BAP permitió la multiplicación y elongación de los brotes, aspecto que coincide con lo señalado por Del Ponte et al. (2001) que ha indicado que el uso de este regulador es común en las especies de Eucalyptus para inducir la proliferación de los explantes.

La aplicación de dosis crecientes de AIB para inducir rizogénesis in vitro permitió que con la utilización de $39 \mu \mathrm{M}$ AIB enraizara el $44 \%$ de brotes cultivados in vitro (Figura $\mathrm{N}^{\circ} 2$ ), en un periodo de 15 días. Concentraciones superiores del regulador disminuyeron la respuesta rizogénica. Los resultados obtenidos difieren de lo señalado por Alfenas et al. (2004) quienes indican que una dosis de 4,87 $\mu \mathrm{M}$ AIB permite la rizogénesis en Eucalyptus en un periodo de 7 a 15 días.

Los mayores porcentajes de enraizamiento se lograron en las cruzas $\mathrm{B}$ y $\mathrm{E}$ en relación al material vegetal restante (Figura $\left.N^{\circ} 4\right)$. La disminución en el contenido de macronutrientes en el medio MS (1962) a $1 / 4$ de concentración permitió obtener raíces largas y gruesas (Figura $N^{\circ} 3 \mathrm{D}$ ) lo que coincide con lo señalado por Mokotedi et al. (2000). La aplicación de $39 \mu \mathrm{M}$ AIB permitió enraizar un $60 \%$ de los brotes de la cruza B y un $51,2 \%$ de los brotes de la cruza $E$, el enraizamiento logrado coincide con lo señalado por Gomes y Canhoto (2003), sin embargo la dosis utilizada del regulador (AIB) supera a la señalada por dichos autores (14,7 $\mu \mathrm{M}$ AIB), concentración que también fue aplicada, pero que indujo sólo un $21 \%$ de rizogénesis. Además del aumento en la dosis de AIB empleada, el aumento en la intensidad luminosa durante el proceso de rizogénesis podría haber facilitado la traslocación de sinergistas auxínicos (fenoles) y carbohidratos a la base de los tallos, favoreciendo la diferenciación de raíces, aspecto que ha sido mencionado por Wang (1992).

Mokotedi et al. (2000) logran el enraizamiento in vitro de dos clones de un híbrido Eucalyptus grandis Hill ex Maid. x E. nitens disminuyendo los macronutrientes del medio MS (1962) a 1/4 concentración de los macronutrientes para un $75 \%$ de enraizamiento en un clon con 0,5 UM AIB. La disminución en el contenido de macronutrientes del medio MS (1962) permitió alcanzar hasta un $100 \%$ de enraizamiento in vitro en clones de la cruza B (Figura $N^{\circ} 5$ ), coincidiendo con lo señalado por dichos autores. Para otros genotipos de la misma cruza, no se logró el enraizamiento.

Alfenas et al. (2004) han indicado que altas concentraciones de BAP utilizadas en el medio de multiplicación afectarían el desarrollo rizogénico posterior, concentraciones de $0,3 \mu \mathrm{M}$ BAP junto a 0,5 $\mu \mathrm{M}$ ANA serían suficientes para multiplicar y elongar brotes en Eucalyptus. En esta investigación se utilizó concentraciones de 1,1 $\mu \mathrm{M}$ BAP y 0,05 $\mu \mathrm{M}$ ANA, que permitieron multiplicar y elongar los brotes, sin embargo, la acumulación de BAP en los tejidos podría estar disminuyendo el proceso de enraizamiento en dichos genotipos.

\section{CONCLUSIONES}

En esta investigación se concluye que las condiciones ambientales de $23^{\circ} \mathrm{C}$ favorecieron una germinación sobre el $50 \%$ para las semillas control y cruzas A, B, C, F, E, obteniéndose solo un $40 \%$ de germinación en las semillas de la cruza $\mathrm{E}$.

La fuerza iónica del medio de cultivo afectó la proliferación de 63 genotipos, los cuales no prosperaron en el medio de multiplicación, logrando multiplicar solo 140 clones.

La disminución en la fuerza iónica del medio de cultivo permitió diferenciar raíces largas y gruesas, la adición de $39 \mu \mathrm{M}$ AIB indujo un $60 \%$ de brotes enraizados en la cruza B y un $51,2 \%$ en la cruza $\mathrm{E}$, respuestas rizogénicas superiores en relación al resto de los cruzamientos y control. 
En el cruzamiento B, existió variabilidad en el enraizamiento de los clones, con un $100 \%$ de enraizamiento de un clon a ausencia de enraizamiento en otros.

\section{REFERENCIAS}

Alfenas, A. C.; R. G. Mafia y T. F. de Assis, 2004. Clonagem e doencas do eucalipto. Vicosa: UFV. 442p.

Chaisurisri, K., D. G. Edwards y Y. A. El Kassaby, 1992. Genetic control of seed size and germination in Sitka Spruce. Silvae Genet. 41: 348-355.

De Fossard, R. A. 1974. Tissue culture of Eucalyptus. Austr. For. 37: 43-54.

Del Ponte, E. M.; V .L. Mattei; J. A. Peters y T. F. de Assis, 2001. Multiplicacao e enraizamento in vitro de Eucalyptus globulus subsp. globulus Labill. Revista Arvore, Vicosa. 25 (1): 1-8.

Dutra, L. F.; Wendling, I. y Brondani. G. E., 2009. A micropropagacao de Eucalipto. Pesquisa Florestal Brasileira, Colombo. $58: 49-59$

Eldridge, K.; Davidson, J.; Harwood, C. and Van Wyk, G., 1993. Eucalypt domestication and breeding. Clarendon Press, Oxford.

Gomes, F. y Canhoto, J. M., 2003. Micropropagation of Eucalyptus nitens Maiden (Shining Gum). In Vitro Cellular \& Developmental Biology Plant. 39. (3): 316-321.

Humara, J. M.; López, M.; Casares, A. y Majada, J., 2000. Temperature and provenance as two factors affecting Eucalyptus nitens seed germination. Forestry. 73 (1): 87-90.

Mokotedi, O. M. E.; Watt, M. P.; Pammenter, N. W. y Blakeway, F. C., 2000. In vitro rooting and subsequent survival of two clones of a cold-tolerant Eucalyptus grandis $x$ E. nitens hybrid. Hortscience. 35 (6): 1163-1165.

Murashige, T. and Skoog, F., 1962. A revised medium for rapid growth and bioassays with Tobacco tissue cultures. Physiol. Plant. 15:473-497.

Taylor, J. P.; Wester, D. B. and Smith, L. M., 1999. Soil disturbance, flood management, and riparian woody plant establishment in the Rio Grande floodplain. Wetlands 19: 372-382.

Wang, Q., 1992. The effect of light, darkness and temperatura on micropropagation of the pear rootstock BP10030. Journal Horticultural Science. 676: 869-876.

Williams, J. E. and Woinarski, J. C. Z., 1997. Eucalypt Ecology: Individuals to Ecosystems. Cambridge University Press, Cambridge, UK. 
